\title{
WITT GROUP OF HERMITIAN FORMS OVER A NONCOMMUTATIVE DISCRETE VALUATION RING
}

\author{
L. OUKHTITE
}

Received 16 June 2004 and in revised form 28 February 2005

We investigate Hermitian forms on finitely generated torsion modules over a noncommutative discrete valuation ring. We also give some results for lattices, which still are satisfied even if the base ring is not commutative. Moreover, for a noncommutative discrete-valued division algebra $D$ with valuation ring $R$ and residual division algebra $\bar{D}$, we prove that $W(\bar{D}) \cong W T(R)$, where $W T(R)$ denotes the Witt group of regular Hermitian forms on finitely generated torsion $R$-modules.

\section{Introduction}

There has been considerable interest in Witt group of bilinear forms over commutative rings. In particular, several authors are interested in commutative discrete valuation rings and it was clear that a similar theory could be developed in noncommutative settings. Therefore, most of the topics of this paper are generalizations of known results on Hermitian forms and Witt group over commutative discrete valuation rings. In Theorem 2.7, we present useful results on lattices and Hermitian forms which are a straightforward generalization of the commutative situation. For a commutative discrete valuation ring $R$ with residual field $k$, it is well known that $W(k) \cong W T(R)$, where $W T(R)$ denotes the Witt group of symmetric regular bilinear forms on finitely generated torsion $R$-modules (see, e.g., [4, Theorem 2.1, page 207]). In Theorem 3.3, we extend this result to noncommutative case. More precisely, for a discrete-valued division algebra $D$ with valuation ring $R$ and residual division algebra $\bar{D}$, we prove that $W(\bar{D}) \cong W T(R)$. To conclude this paper, Theorem 3.6 explores an exact sequence which is known only for Dedekind domain.

Let $D$ be a noncommutative finite-dimensional central division algebra over a field $F$ and let $\star$ be an involution of the first kind on $D$, that is, $\star$ leaves $F$ elementwise invariant. For clarity, it is interesting to elucidate some of the terminology to be used. If $v$ is a discrete valuation on $D$ with valuation ring $R$ and if $M$ denotes the maximal ideal of $R$, then $\bar{D}:=R / M$ is called the residual division algebra. Since every element $x \in D$ satisfies $x \in R$ or $x^{-1} \in R$, then every ideal of $R$ is a two-sided ideal. Indeed, if $\pi$ is a prime element of $R$, that is, a generator of $\mathcal{M}$, and if $I$ is a one-sided ideal of $R$, then $I=\pi^{n} R=R \pi^{n}$, 
where $n=\inf \{v(x) / x \in I\}$. In particular, $R$ is then a principal ideal domain. As $a R=R a$ for all $a \in R$, then elementary divisor theorem holds even if $R$ is not commutative. In all that follows, $\mathcal{U}(R)$ will stand for the set of all invertible elements of $R$. Recall that if $V$ is a $D$-vector space with dimension $n$, then a submodule $L$ of $V$ is called an $R$-lattice (or simply lattice) on $V$ if $L=\oplus_{i=1}^{n} R e_{i}$ for some basis $\left(e_{1}, \ldots, e_{n}\right)$ of $V$. Moreover, if $h$ is a Hermitian form on $V$, then the dual lattice of $L$ is defined by $L^{\#}:=\{x \in V / h(x, L) \subset R\}$. Finally, an $R$-module $J \subset D$ is said to be a fractional ideal if there exists $a \in R$ such that $a J \subset R$. Furthermore, if $I J=J I=R$ for some fractional ideal $I$, then $J$ is invertible with inverse $I$. In particular, for $a \in R, a R$ is an invertible fractional ideal with inverse $a^{-1} R=$ $R a^{-1}$. Moreover, if we set $[I: R]:=\{x \in D / x I \subset R\}$, then $I^{-1}=[I: R]$.

\section{Hermitian forms and lattices}

In this section, we continue with the same notations as in the preceding section. For a finitely generated torsion left $R$-module $M$, its dual $M^{*}:=\operatorname{Hom}_{R}(M, T=D / R)$ is endowed with a natural right $R$-module structure defined by $f \cdot r(m)=f(m) r$ for $f \in M^{*}$, $r \in R$, and $m \in M$.

Lemma 2.1. The restriction of $\star$ to $R$ is an involution. Moreover, $\star$ induces an involution:

(1) $\overline{\text { on }} \overline{\mathrm{D}}$ defined by $\bar{x}^{\bar{\star}}:=\bar{x}^{\bar{\star}}$;

(2) $\star^{\star}$ on $D / R$ defined by $(d+R)^{\star}:=d^{\star}+R$.

Proof. From theory of valued division algebras, it is well known that the restriction of $v$ to the field $F$ extends in a unique way to $D$, moreover, this extension is $v$. To prove the restriction of $\star$ to $R$ is an involution, it suffices to show that $v(x)=v\left(x^{\star}\right)$ for all $x \in R$. For $x \in D$, setting $\omega(x):=v\left(x^{\star}\right)$, then it is straightforward to check that $\omega$ is a valuation on $D$. Since $\star$ is an involution of the first kind, we deduce that $\omega / F=v / F$, hence $\omega=v$ so that $v(x)=v\left(x^{*}\right)$ for all $x \in R$. Consequently, (1) and (2) hold.

Since $\star$ is an involution on $R$, then $M^{*}$ can again be regarded as a left module if one defines $r \cdot f(m)=f(m) r^{*}$.

LEMMA 2.2. $M \cong M^{*}$, and $M$ is canonically isomorphic to $M^{* *}$.

Proof. For the nontrivial part, as $R$ is in particular a left principal ring satisfying $a R=R a$ for all $a \in R$, there exists an ascending sequence $\left(I_{1}, \ldots, I_{n}\right)$ of ideals of $R$ such that $M \cong$ $\oplus_{i=1}^{n} R / I_{i}$. Since $(M \oplus N)^{*}=M^{*} \oplus N^{*}$, it then suffices to prove the lemma for $M=R / I$. On the other hand, for each homomorphism $f \in M^{*}$, we have $f(\bar{r})=r f(\overline{1})$ for all $r \in R$. Setting $\bar{\beta}=f(\overline{1})$, we then get $0=f(\bar{x})=x \beta+R$ for all $x \in I$, which yields $x \beta \in R$ so that $\beta \in\{d \in D / I d \subset R\}=\{d \in D / d I \subset R\}=I^{-1}$. Thus $M^{*} \cong I^{-1} / R$. From $R$ principal, we deduce $I=a R=R a$ for some $a \in R$, therefore $M^{*} \cong I^{-1} / R=a^{-1} R / R \cong R / a R=M$.

A Hermitian form on $M$ is a biadditive map $h: M \times M \rightarrow D / R$ such that, for $x, y \in M$ and $r, \beta \in R: h(r x, \beta y)=r h(x, y) \beta^{*}, h(x, y)=h(y, x)^{*}$. Furthermore, $h$ is called regular if $\hat{h}: M \rightarrow M^{*}$ defined by $\hat{h}(x)=h(x, \cdot)$ is an isomorphism of $R$-modules. For a submodule $N$ of $M$, we define $N^{\perp}=\{x \in M / h(x, n)=0 \forall n \in N\}$. In view of regularity of $h$, we obtain $\left(N^{\perp}\right)^{\perp}=N$. Recall that $(M, h)$ is called weakly metabolic if there exists a submodule $N$ of $M$ such that $N=N^{\perp}$. 
In the sequel, $W T(R)$ denotes the Witt group of regular Hermitian forms on finitely generated torsion $R$-modules, where weakly metabolic forms are identified with 0 .

Proposition 2.3. Let $N$ be a submodule of $(M, h)$ with $N \subset N^{\perp}$. Then $h$ induces a regular Hermitian form $\bar{h}$ on $N^{\perp} / N$ such that $(M, h) \oplus\left(N^{\perp} / N,-\bar{h}\right)$ is weakly metabolic.

Proof. Consider the map $\bar{h}: N^{\perp} / N \times N^{\perp} / N \rightarrow D / R$ defined by $\bar{h}(\bar{x}, \bar{y})=h(x, y)$. If $x, x^{\prime}$, $y, y^{\prime}$ are elements in $N^{\perp}$ such that $(\bar{x}, \bar{y})=\left(\bar{x}^{\prime}, \bar{y}^{\prime}\right)$, then $x=x^{\prime}+n$ and $y=y^{\prime}+n^{\prime}$, where $n, n^{\prime} \in N$. Using $N \subset N^{\perp}$, we then get $\bar{h}(\bar{x}, \bar{y})=h\left(x^{\prime}+n, y^{\prime}+n^{\prime}\right)=h\left(x^{\prime}, y^{\prime}\right)$ so that $\bar{h}$ is a well-defined map. Moreover, for $x, y \in N^{\perp}$, we have $\bar{h}(\alpha \bar{x}, \beta \bar{y})=\bar{h}(\overline{\alpha x}, \overline{\beta y})=\alpha h(x, y) \beta^{*}=$ $\alpha \bar{h}(\bar{x}, \bar{y}) \beta^{*}$ for all $\alpha, \beta \in R$. For regularity of $\bar{h}$, if $x \in N^{\perp}$ is such that $\bar{h}(\bar{x}, \bar{y})=0$ for all $y \in N^{\perp}$, then $h(x, y)=0$ for all $y \in N^{\perp}$. Consequently, $x \in\left(N^{\perp}\right)^{\perp}=N$ and then $\bar{x}=0$. Let $f \in\left(N^{\perp} / N\right)^{*}$, then $f \circ \pi \in\left(N^{\perp}\right)^{*}$, where $\pi: N^{\perp} \rightarrow N^{\perp} / N$ is the canonical projection. The surjectivity of $M \stackrel{\hat{h}}{\rightarrow} M^{*} \rightarrow\left(N^{\perp}\right)^{*}$ assures existence of $x \in M$ such that $f(\bar{y})=f(y+$ $N)=h(x, y)$ for all $y \in N^{\perp}$. In particular, for $y \in N, f(\bar{y})=0=h(x, y)$, thus $x \in N^{\perp}$ and $f=\hat{\bar{h}}(x)$, which proves the regularity of $\bar{h}$. Finally, the submodule $\Delta=\left\{(x, \bar{x}) / x \in N^{\perp}\right\}$ of $M \oplus N^{\perp} / N$ satisfies $\Delta^{\perp}=\Delta$. Thus $(M, h) \oplus\left(N^{\perp} / N,-\bar{h}\right)$ is weakly metabolic.

In the classical theory of regular symmetric bilinear forms over a commutative field $F$, orthogonal basis plays a key role in studying the Witt group $W(F)$. Furthermore, existence of orthogonal basis is proved in noncommutative case (see [1, Theorem 3, page 153]). In our situation, we can give a short proof for this result as follows.

Proposition 2.4. Every regular Hermitian space $(V, h)$ has an orthogonal basis.

Proof. Suppose $h \neq 0$, otherwise each basis is clearly orthogonal. There exist $x, y \in V$ such that $h(x, y)=1$. If $h$ satisfies $h(z, z)=0$ for all $z \in V$, then $h(x+\alpha y, x+\alpha y)=0=\alpha+\alpha^{*}$ so that $\alpha^{*}=-\alpha$ for all $\alpha \in D$, contradicting $1^{*}=1$. Thus there exists $z \in V$ for which $h(z, z) \neq 0$ and $h(z, z)$ is then invertible in $D$. The regularity of $D z$ yields $V=D z \perp(D z)^{\perp}$. Reasoning by induction, this completes the proof.

Remark 2.5. As an application of the previous result, every regular Hermitian space contains a lattice $L$ such that $L \subset L^{\#}$. Indeed, let $L=\oplus_{i=1}^{n} R e_{i}$ be a lattice on $(V, h)$ and let $\left(e_{i}\right)_{1 \leq i \leq n}$ be an orthogonal basis of $V$. Hence, $h\left(e_{i}, e_{j}\right)=0$ for $i \neq j$ and $h\left(e_{i}, e_{i}\right)=\lambda_{i} \in D$ and either $\lambda_{i} \in R$ or $\lambda_{i}^{-1} \in R$. Assume $\lambda_{i}^{-1} \in R$ for some $1 \leq i \leq n$, then $\left(e_{1}, \ldots, \lambda_{i}^{-1} e_{i}, \ldots, e_{n}\right)$ is an orthogonal basis of $V$ satisfying $h\left(e_{j}, e_{j}\right)=\lambda_{j} \in R$ for $j \neq i$ and $h\left(\lambda_{i}^{-1} e_{i}, \lambda_{i}^{-1} e_{i}\right)=$ $\left(\lambda_{i}^{*}\right)^{-1} \in R$, accordingly, $L \subset L^{\#}$. This remark will be used in the sequel.

The following lemma describes relationship between linear $R$-independence and linear $D$-independence for elements in $V$.

Lemma 2.6. Let $e_{1}, \ldots, e_{n}$ be elements in $V$, then $e_{1}, \ldots, e_{n}$ are linearly $R$-independent if and only if $e_{1}, \ldots, e_{n}$ are linearly $D$-independent.

Proof. For the nontrivial sense, suppose $e_{1}, \ldots, e_{n}$ are not linearly $D$-independent, then there exist $d_{1}, \ldots, d_{m} \in D-\{0\}(m \leq n)$ such that $\sum_{i=1}^{m} d_{i} e_{i}=0$.

Let $v\left(d_{s}\right)=\inf \left\{v\left(d_{i}\right) / 1 \leq i \leq n\right\}$, then for all $1 \leq i \leq m$, we have $d_{i}=r_{i} d_{s}$ for some $r_{i} \in$ $R-\{0\}$. Accordingly, $\sum_{i=1}^{m} r_{i} d_{s} e_{i}=0$ so that $\sum_{i=1}^{m} d_{s}^{-1} r_{i} d_{s} e_{i}=0$ proving our proposition, since $d_{s}^{-1} r_{i} d_{s} \in R-\{0\}$. 
In the following theorem, we collect some useful results on lattices. Our approach is based on commutative case with different proof due to the use of valuation.

Theorem 2.7. Let $L_{1}$ and $L_{2}$ be two lattices on $V$. Then

(1) there exist $a, b \in R$ such that $a L_{1} \subset L_{2}$ and $b L_{2} \subset L_{1}$,

(2) there exist a basis $\left(e_{1}, \ldots, e_{n}\right)$ of $V$ and fractional ideals $I_{1}, \ldots, I_{n}$ such that $L_{1}=$ $\oplus_{i=1}^{n} R e_{i}$ and $L_{2}=\oplus_{i=1}^{n} I_{i} e_{i}$,

(3) $\left(L_{1} \cap L_{2}\right)^{\#}=L_{1}^{\#}+L_{2}^{\#}$ and $\left(L_{1}+L_{2}\right)^{\#}=L_{1}^{\#} \cap L_{2}^{\#}$,

(4) if $L_{1} \subset L_{2}$, then $L_{2}^{\#} \subset L_{1}^{\#}$.

Proof. (1) Writing $L_{1}=\oplus_{i=1}^{n} R e_{i}$ and $L_{2}=\oplus_{i=1}^{n} R f_{i}$ where both $\left(e_{1}, \ldots, e_{n}\right)$ and $\left(f_{1}, \ldots, f_{n}\right)$ are $D$-bases of $V$. We fix $1 \leq s \leq n$, there exist $d_{s_{1}}, \ldots, d_{s_{n}} \in D$ such that $f_{s}=\oplus_{j=1}^{n} d_{s_{j}} e_{j}$. Consider $v\left(d_{s_{t}}\right)=\inf \left\{v\left(d_{s_{j}}\right) / 1 \leq j \leq n\right\}$, then for all $1 \leq j \leq n$, we have $d_{s_{j}}=r_{j} d_{s_{t}}$, where $r_{j} \in R$. If $d_{s_{t}} \in R$, then $d_{s_{j}} \in R$ for all $j$, otherwise $d_{s_{t}}^{-1} \in R$ so that $d_{s_{t}}^{-1} d_{s_{j}}=d_{s_{t}}^{-1} r_{j} d_{s_{t}} \in R$ for all $j \neq t$ and $d_{s_{t}}^{-1} d_{s_{t}}=1 \in R$ which proves existence of $r_{s} \in R$ such that $r_{s} d_{s_{j}} \in R$ for all $1 \leq j \leq n$. Hence, $r_{s} f_{s} \in L_{1}$ for all $1 \leq s \leq n$. Setting $b=\prod_{i=1}^{n} r_{i} \in R$, we then get $b R f_{i}=$ $r_{1} \cdots r_{i} \cdots r_{n} R r_{i}^{-1} r_{i} f_{i} \subset R r_{i} f_{i} \subset L_{1}$ for all $i$, hence $b L_{2} \subset L_{1}$. A similar reasoning yields $a L_{1} \subset L_{2}$ for some element $a \in A$.

(2) Let $a$ be a nonzero element in $R$ such that $a L_{2} \subset L_{1}$. As $a L_{2}$ is a lattice on $V$, then $a L_{2}$ is a finitely generated free $R$-module with $\operatorname{rank} n=[V: D]$. Applying elementary divisor theorem to the submodule $a L_{2}$ of $L_{1}$, there exist an $R$-basis $\left(e_{1}, \ldots, e_{n}\right)$ of $L_{1}$ and elements $b_{1}, \ldots, b_{n} \in R-\{0\}$ such that $\left(b_{1} e_{1}, \ldots, b_{n} e_{n}\right)$ is an $R$-basis of $a L_{2}$. Therefore, $L_{1}=$ $\oplus_{i=1}^{n} R e_{i}$ and $a L_{2}=\oplus_{i=1}^{n} R b_{i} e_{i}$. Consequently, $L_{2}=\oplus_{i=1}^{n} I_{i} e_{i}$, where $I_{i}=a^{-1} R b_{i}=R a^{-1} b_{i}$ is a fractional ideal. Finally, Lemma 2.6 implies that $\left(e_{1}, \ldots, e_{n}\right)$ is a $D$-basis of $V$.

(3) and (4) are clear.

Remark 2.8. If $L=\oplus_{i=1}^{n} R e_{i}$ is a lattice on a regular Hermitian space, then $\left(L^{\#}\right)^{\#}=L$. Furthermore, it is interesting to note that $L^{\#}=\oplus_{i=1}^{n} R e_{i}^{\prime}$, where $\left(e_{1}^{\prime}, \ldots, e_{n}^{\prime}\right)$ is the dual basis of $\left(e_{1}, \ldots, e_{n}\right)$.

\section{Main results}

From [3, Corollary 1.4.4, page 396], it follows that every finitely generated torsion-free module over a principal ideal domain is a free module. The following proposition gives another proof for this result due to the use of valuation's arguments.

Proposition 3.1. Every finitely generated torsion-free module is a free module.

Proof. Let $\left(m_{1}, \ldots, m_{n}\right)$ be a minimal set of generators of $M$ with $\sum_{i=1}^{n} a_{i} m_{i}=0$ and let $v\left(a_{i}\right)=\inf \left\{v\left(a_{j}\right) / 1 \leq j \leq n\right\}$. For all $j$, we have $v\left(a_{j}\right)=v\left(a_{i}\right)+s_{j}=v\left(a_{i} \pi^{s_{j}}\right)$, and thus $a_{j}=$ $a_{i} \pi^{s_{j}} u_{j}$, where $u_{j}$ is an invertible element of $R$. If $a_{i}=0$, then $a_{j}=0$ for all $j$ which implies that $\left(m_{1}, \ldots, m_{n}\right)$ is free. Assume $a_{i} \neq 0$, as $M$ is torsion-free, the fact that $a_{i}\left(\pi^{s_{1}} u_{1} m_{1}+\right.$ $\left.\cdots+m_{i}+\pi^{s_{n}} u_{n} m_{n}\right)=0$ implies $\pi^{s_{1}} u_{1} m_{1}+\cdots+m_{i}+\pi^{s_{n}} u_{n} m_{n}=0$ so that $\left(m_{1}, \ldots\right.$, $\left.m_{i-1}, m_{i+1}, \ldots, m_{n}\right)$ generated $M$, contradicting minimality of $\left(m_{1}, \ldots, m_{n}\right)$. Accordingly, $\left(m_{1}, \ldots, m_{n}\right)$ is free.

Proposition 3.2. Assume $2 \in \mathcal{U}(R)$. Then every regular Hermitian module $(M, h)$ has an orthogonal basis. 
Proof. Suppose existence of an element $x \in M$ such that $h(x, x) \in \mathcal{U}(R)$. It is easily seen that $R x$ is a regular submodule of $M$, hence $M=N \perp N^{\perp}$. Reasoning by induction, $(M, h)$ admits an orthogonal basis $\left(e_{1}=x, e_{2}, \ldots, e_{n}\right)$.

Now to prove existence of $x \in M$ with $h(x, x) \in \mathcal{U}(R)$, let $x, y \in M$ such that $h(x, y)=$ $r \neq 0$. If $h(z, z)=0$ for all $z \in M$, then $h(x+u y, x+u y)=0$ so that $r u^{*}+u r^{*}=0$ for all $u \in R$. In particular, for $u=1$, we get $r u^{*}=u r$ for all $u \in R$. Consequently, for $u, v \in R$, we then have $u v r=r(u v)^{*}=r v^{*} u^{*}=v r u^{*}=v u r$ which yields $u v=v u$ since $R$ is a domain and therefore contradicts the noncommutativity of $R$. Hence, $M$ contains an element $z$ satisfying $h(z, z) \neq 0$. Furthermore, we claim existence of $x \in M$ such that $h(x, x) \in \mathcal{U}(R)$. Indeed, otherwise $h(x, x) \in M$ for all $x \in M$. Let $\left(e_{1}, \ldots, e_{n}\right)$ denote a basis of $M$ over $R$ and consider the map $\phi: M \rightarrow R$ defined by $\phi\left(e_{i}\right)=\delta_{i 1}$. It is clear that $\phi \in M^{*}$ and the regularity of $h$ implies $\phi=\hat{h}(x)$ with $x \in M$. From $1=\phi\left(e_{1}\right)=h\left(x, e_{1}\right)$, we deduce $2=$ $h\left(x, e_{1}\right)+h\left(e_{1}, x\right) \in M$ which completes the proof.

THEOREM 3.3. $W T(R) \cong W(\bar{D})$ is an isomorphism which depends on the choice of the prime element $\pi$.

Proof. (1) Since the action $r \cdot(d+R)=r d+R$ endows $D / R$ with a left $R$-module structure, the fact that $(R, \star)$ is a ring with involution implies that $D / R$ can be transformed into a right module via $(d+R) r=r^{*} d+R$. On the other hand, if $M$ is a finite-dimensional $\bar{D}$-vector space, then $M$ is a finite generated torsion $R$-module $(\pi \cdot m=0$ for all $m \in M$, $\pi$ the nonzero generator of $\mathcal{M})$. Now, consider a regular Hermitian form $h: M \times M \rightarrow \bar{D}$. For $x, y \in M, \pi^{-1} h(x, y) \in D / R$ and $\pi^{-1} h(x, y)=\pi^{-1} h(x, y) \pi^{*}\left(\pi^{*}\right)^{-1}=h(x, y)\left(\pi^{*}\right)^{-1}$. From $\pi^{-1} h(\alpha x, \beta y)=h(\alpha x, \beta y)\left(\pi^{*}\right)^{-1}=\alpha h(x, \beta y)\left(\pi^{*}\right)^{-1}=\alpha \pi^{-1} h(x, \beta y)=\alpha \pi^{-1} h(x, y) \beta^{*}$ and $\pi^{-1} h(y, x)=\pi^{-1} h(x, y)^{*}=h(x, y)^{*}\left(\pi^{*}\right)^{-1}=\left(\pi^{-1} h(x, y)\right)^{*}$ for all $\alpha, \beta \in R$, it follows that $\pi^{-1} h$ is a Hermitian form. To prove regularity of $\pi^{-1} h$, suppose $\pi^{-1} h(x, y)=0$ for all $y \in M$, then $h(x, y) \in \pi R$ in such a way that $x=0$. Moreover, if $f: M \rightarrow D / R$, then $f(\pi x)=\pi f(x)=0$ for all $x \in M$, and thus $\pi f(x) \in R$. From $M \stackrel{\pi f}{\rightarrow} R \stackrel{s}{\rightarrow} R / \pi R$, the regularity of $h$ then assures existence of $x \in M$ such that $s \circ \pi f=\hat{h}(x)$, that is, $\pi f(y)+\pi R=$ $h(x, y)$ for all $y \in M$. Therefore, $f(y)=\pi^{-1} h(x, \cdot)(y)$ proving that

$$
\Psi: W(\bar{D}) \longrightarrow W T(R), \quad(V, h) \longmapsto\left(V, \pi^{-1} h\right)
$$

is a well-defined map.

(2) Let $M$ be a finitely generated torsion $R$-module and let $h: M \times M \rightarrow D / R$ be a regular Hermitian form. Hence, for $\alpha, \beta \in R, h(\alpha x, \beta y)=\alpha h(x, y) \beta^{*}=\beta \alpha h(x, y)=h(\alpha \beta x, y)$ for all $x, y \in M$. Then we have to distinguish two cases.

(i) $\pi M=\{0\}$. It is readily verified that $M$ is a left $\bar{D}$-vector space by the action $\bar{r} \cdot m=r m$. In view of $\pi h(\alpha x, \beta y)=h(\alpha x, \beta y) \pi^{*}=\alpha h(x, \beta y) \pi^{*}=\alpha \pi h(x, \beta y)=$ $\alpha \pi h(x, y) \beta^{*}$, we deduce that $\pi h: M \times M \rightarrow \bar{D}$ is a Hermitian form. To prove regularity of $\pi h$, let $x \in M$ be such that $\pi h(x, y)=0$ for all $y \in M$, then $h(x, y) \in$ $R$ and the regularity of $h$ yields $x=0$. Furthermore, if $f: M \rightarrow R / \pi R$, then from $\pi^{-1} f(\alpha x)=\pi^{-1} f(\alpha x) \pi^{*}\left(\pi^{*}\right)^{-1}=\left(\pi^{-1} f(\alpha x) \pi^{*}\right)\left(\pi^{*}\right)^{-1}=f(\alpha x)\left(\pi^{*}\right)^{-1}=$ $\alpha f(x)\left(\pi^{*}\right)^{-1}=\alpha \pi \pi^{-1} f(x)\left(\pi^{*}\right)^{-1}=\alpha \pi\left(\pi^{-1} f(x)\left(\pi^{*}\right)^{-1}\right)=\alpha \pi\left(\pi^{2} f(x)\right)=$ $\alpha \pi^{-1} f(x)$, we get $\pi^{-1} f \in \operatorname{Hom}_{R}(M, D / R)$ so that $\pi^{-1} f=\hat{h}(x)$ for some $x \in M$. Thus $f=\widehat{\pi h}(x)$ proving regularity of $\pi h$ in this case. 
(ii) $\pi^{k} M=\{0\}$ and $\pi^{k-1} M=M_{1} \neq\{0\}$ for some $k \geq 2$. The fact that $h(\alpha x, \beta y)=$ $h(\beta \alpha x, y)$ then yields $h\left(\pi^{k-1} x, \pi^{k-1} y\right)=h\left(\pi^{2(k-1)} x, y\right)=0$, and therefore $M_{1} \subset$ $M_{1}^{\perp}$. Setting $\overline{M_{1}}=M_{1}^{\perp} / M_{1}$ and applying Proposition 2.3 , we get $(M, h)=\left(\overline{M_{1}}, \bar{h}\right)$ in $W T(R)$. Let $h_{1}=\pi h$ and $M_{2}=\pi^{k-1} M_{1}$, then clearly $M_{2} \subset M_{2}^{\perp}$. Furthermore, $\pi^{k-1} \overline{M_{1}}=\{0\}$ because $\pi^{k-1} M_{1}^{\perp} \subset \pi^{k-1} M=M_{1}$. We then construct $\left(\overline{M_{r}}, \bar{h}\right)$ such that $(M, h)=\left(\overline{M_{r}}, \bar{h}\right)$ in $W T(R)$ and $\pi \overline{M_{r}}=\{0\}$. Therefore, $\left(\overline{M_{r}}, \pi \bar{h}\right) \in W(\bar{D})$. Consequently,

$$
\begin{aligned}
\Phi: W T(R) & \longrightarrow W(\bar{D}), \\
(M, h) & \longmapsto\left(\overline{M_{r}}, \pi \bar{h}\right)
\end{aligned}
$$

is a well-defined map. Moreover, $\Phi \circ \Psi=\operatorname{Id}_{W(\bar{D})}$ and $\Psi \circ \Phi=\operatorname{Id}_{W T(R)}$.

Let $L$ be a finitely generated projective $R$-module with rank $n$. It is clear that $L \otimes_{R} D$ is a right $D$-vector space with dimension $n$. Moreover, every Hermitian form $h: L \times L \rightarrow R$ induces a Hermitian form $h_{L}$ on $L \otimes_{R} D$ defined by $h_{L}\left(x \otimes d, y \otimes d^{\prime}\right)=d^{*} h(x, y) d^{\prime}$.

LEMma 3.4. With the notations above, if $h$ is regular, then $h_{L}$ is regular too.

Proof. Let $\left\{e_{i}\right\}_{1 \leq i \leq n}$ be an $R$-basis of $L$ and let $\left\{e_{i}^{\prime}\right\}_{1 \leq i \leq n}$ denote its dual basis defined by $h\left(e_{i}, e_{i}^{\prime}\right)=\delta_{i j}$. It is easy to verify that $\left\{e_{i} \otimes 1\right\}_{1 \leq i \leq n}$ is a $D$-basis of $L \otimes D$ with dual basis $\left\{e_{i}^{\prime} \otimes 1\right\}_{1 \leq i \leq n}$. Let $x=\sum_{i=1}^{n} e_{i} \otimes d_{i} \in L \otimes D$ such that $h_{L}(x, y)=0$ for all $y \in L \otimes D$, hence $h_{L}\left(x, e_{j}^{\prime} \otimes 1\right)=0=\sum_{i=1}^{n} d_{i}^{*} \delta_{i j}=d_{j}^{*}$ for all $j$ so that $x=0$, thus $h_{L}$ is regular.

Lemma 3.5. Let $(V, h)$ be a Hermitian form and let $L$ be a lattice on $V$ with $L \subset L^{\#}$. Then $\left(L^{\#} / L, \bar{h}\right) \in W T(R)$ does not depend on the choice of the lattice $L$.

Proof. Let $L_{0}$ be a lattice on $V$ such that $L_{0} \subset L$. Using Theorem 2.7, we obtain $L_{0} \subset$ $L \subset L^{\#} \subset L_{0}^{\#}$. Moreover, it is clear that $L / L_{0}$ is a torsion submodule of $L_{0}^{\#} / L_{0}$ which satisfies $\left(L / L_{0}\right)^{\perp}=\left\{x+L_{0}, x \in L_{0}^{\#} / h(x, y) \in R\right.$ for all $\left.y \in L\right\}=L_{0}^{\#} \cap L^{\#} / L_{0}=L^{\#} / L_{0}$ so that $L / L_{0} \subset\left(L / L_{0}\right)^{\perp}$. From Proposition 2.3 , we then get $\left(L_{0}^{\#} / L, h_{L_{0}}\right)=\left(\left(L^{\#} / L_{0}\right) /\left(L / L_{0}\right), \bar{h}_{L_{0}}\right)=$ $\left(L^{\#} / L, h_{L}\right)$ in $W T(R)$. Now, consider two lattices $L_{1}$ and $L_{2}$ on $V$ and set $L_{0}=L_{1} \cap L_{2}$, then $L_{0} \subset L_{1}$ and $L_{0} \subset L_{2}$. Moreover, $\left(L_{0}^{\#} / L_{0}, h_{L_{0}}\right)=\left(L_{1}^{\#} / L_{1}, h_{L_{1}}\right)=\left(L_{2}^{\#} / L_{2}, h_{L_{2}}\right)$.

A regular Hermitian $R$-module $(M, h)$ is called metabolic if there exists a direct summand $N$ of $M$ satisfying $N^{\perp}=N$. However, it suffices that $M$ admits a submodule $N$ such that $N^{\perp}=N$ (see [2, Lemma 1.2, page 122]). In what follows, $W(R)$ denotes the Witt group of regular Hermitian forms on finitely generated projective R-modules, where metabolic modules are identified with 0 .

Recall that if $R$ is a Dedekind domain with quotient field $K$, then the sequence

$$
0 \longrightarrow W(R) \stackrel{i}{\rightarrow} W(D) \stackrel{\delta}{\longrightarrow} W T(R)
$$

defined by $i(L, h)=\left(L \otimes K, h_{K}\right)$ and $\delta(V, h)=\left(L^{\#} / L, \bar{h}\right)$ is exact. The aim of the following theorem is to extend this result to noncommutative discrete valuation ring. 
THeorem 3.6. With the hypothesis above, we have the following exact sequence:

$$
\begin{gathered}
0 \longrightarrow W(R) \stackrel{i}{\longrightarrow} W(D) \stackrel{\delta}{\longrightarrow} W T(R), \\
(L, h) \longmapsto\left(L \otimes D, h_{L}\right), \\
(V, h) \longmapsto\left(\frac{L^{\#}}{L}, \bar{h}\right) .
\end{gathered}
$$

Proof. Concerning the injectivity of $i$, suppose $\left(L \otimes D, h_{L}\right)$ is a hyperbolic space, then there is a $D$-subspace $W$ of $V=L \otimes D$ such that $W=W^{\perp}$. If $\left\{e_{1}, \ldots, e_{2 n}\right\}$ denotes an $R$-basis of $L$, then $\left\{e_{i} \otimes 1\right\}_{i}$ is a $D$-basis of $V$ and we can assume $\left(e_{1} \otimes 1, \ldots, e_{n} \otimes 1\right)$ is a $D$-basis of $W$. Setting $M=\oplus_{i=1}^{n} R e_{i}$, from $h_{L}\left(e_{i} \otimes 1, e_{j} \otimes 1\right)=0$, we have $h\left(e_{i}, e_{j}\right)=0$ for all $i, j$ so that $M \subset M^{\perp}$. Reciprocally, let $x=\sum_{i=1}^{2 n} r_{i} e_{i} \in M^{\perp}$, hence $0=h\left(x, e_{j}\right)=$ $\sum_{i=1}^{2 n} r_{i} h\left(e_{i}, e_{j}\right)=h_{L}\left(\sum_{i=1}^{2 n} e_{i} \otimes r_{i}, e_{j} \otimes 1\right)$ for all $1 \leq j \leq n$. As $\sum_{i=1}^{n} h_{L}\left(e_{i} \otimes r_{i}, e_{j} \otimes 1\right)=0$, then $h_{L}\left(\sum_{i=n+1}^{2 n} e_{i} \otimes r_{i}, e_{j} \otimes 1\right)=0$ for all $1 \leq j \leq n$ in such a way that $\sum_{i=n+1}^{2 n} e_{i} \otimes r_{i} \in W^{\perp}=$ $W=\oplus_{i=1}^{n}\left(e_{i} \otimes 1\right) D$, therefore $\sum_{i=n+1}^{2 n} e_{i} \otimes r_{i}=0$ and thus $r_{i}=0$ for all $n+1 \leq i \leq 2 n$. Hence, $x=\sum_{i=1}^{n} r_{i} e_{i} \in M$ and $M=M^{\perp}$. From [2, Lemma 1.2, page 122], it then follows that $(L, h)$ is metabolic.

To prove $\operatorname{Im} i \subset \operatorname{ker} \delta$, observe that $L$ is a lattice on $V=L \otimes D$ which satisfies $L=L^{\#}$. Indeed, let $\left(e_{i}\right)_{1 \leq i \leq n}$ be an $R$-basis of $L$ with dual basis $\left(e_{i}^{\prime}\right)_{1 \leq i \leq n}$. If $x=\sum_{i} e_{i} \otimes d_{i} \in L^{\#}$, then $h\left(x, e_{j}^{\prime} \otimes 1\right)=d_{j} \in R$ for all $j$. As $L \hookrightarrow L \otimes D$, then $L=L^{\#}$, and therefore $\delta \circ i(L, h)=0$. Conversely, if $\delta(V, h)=0$, then there exists a lattice $L$ on $V$ such that $\left(L^{\#} / L, \bar{h}\right)$ is weakly metabolic. Hence, there is an $R$-module $N$ with $L \subset N \subset L^{\#}$ and $N / L=(N / L)^{\perp}$. Then $(N / L)^{\perp}=N^{\#} \cap L^{\#} / L=N^{\#} / L$ so that $N=N^{\#}$. Thus $V=N \otimes_{R} D$ and $(V, h)=i(N, h / N)$.

\section{References}

[1] N. Jacobson, Lectures in Abstract Algebra. Vol. II: Linear Algebra, Graduate Texts in Mathematics, no. 31, Springer, New York, 1975.

[2] M. Knebusch, A. Rosenberg, and R. Ware, Structure of Witt rings and quotients of Abelian group rings, Amer. J. Math. 94 (1972), 119-155.

[3] M. Knus, Quadratic and Hermitian Forms Over Rings, Grundlehren der Mathematischen Wissenschaften, vol. 294, Springer, Berlin, 1991.

[4] W. Scharlau, Quadratic and Hermitian Forms, Grundlehren der Mathematischen Wissenschaften, vol. 270, Springer, Berlin, 1985.

L. Oukhtite: Département de Mathématiques, Faculté des Sciences et Techniques, Université Moulay Ismaïl, B. P. 509 Boutalamine, 52000 Errachidia, Morocco

E-mail address: oukhtite@math.net 


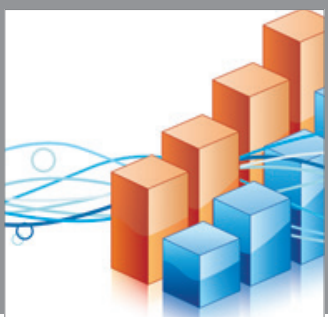

Advances in

Operations Research

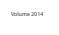

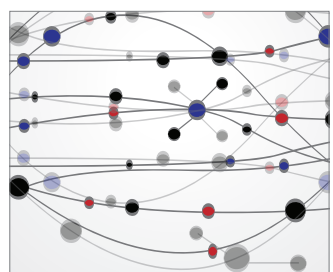

\section{The Scientific} World Journal
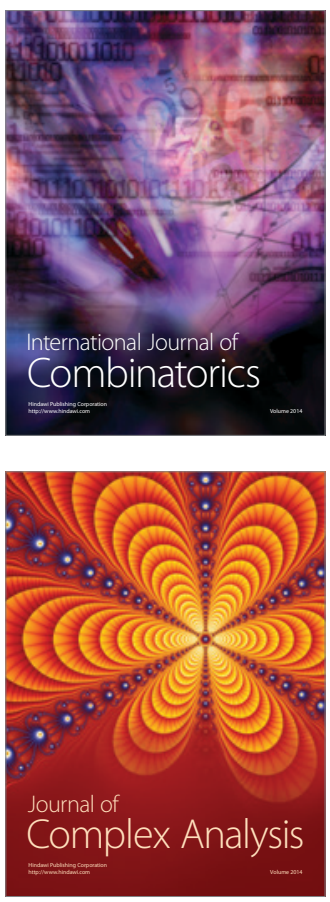

International Journal of

Mathematics and

Mathematical

Sciences
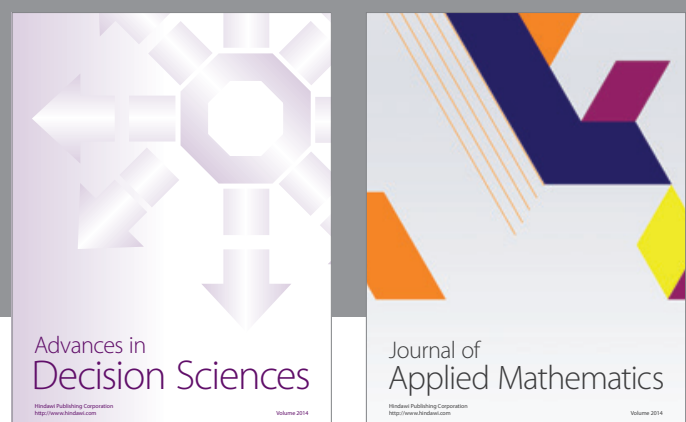

Journal of

Applied Mathematics
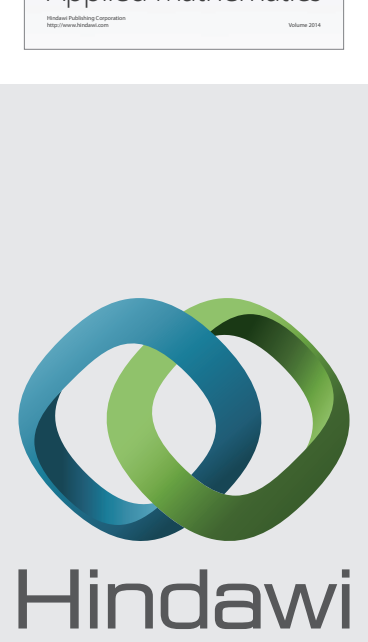

Submit your manuscripts at http://www.hindawi.com
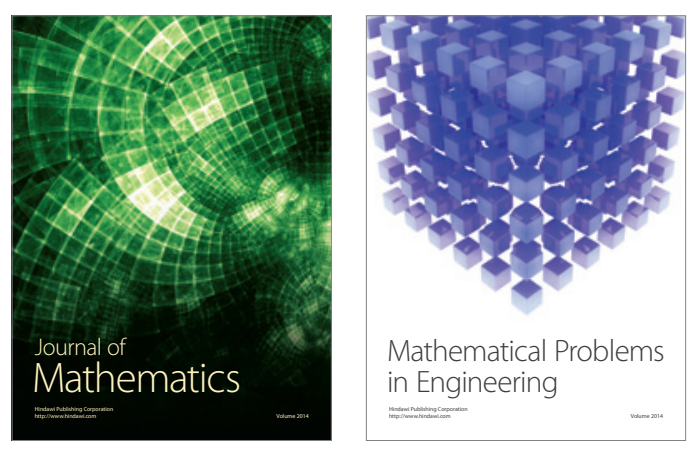

Mathematical Problems in Engineering
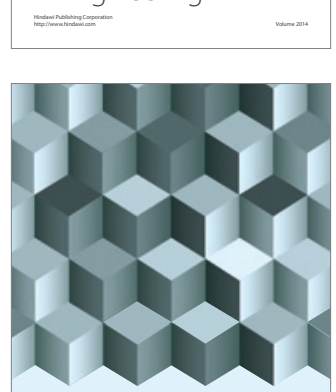

Journal of

Function Spaces
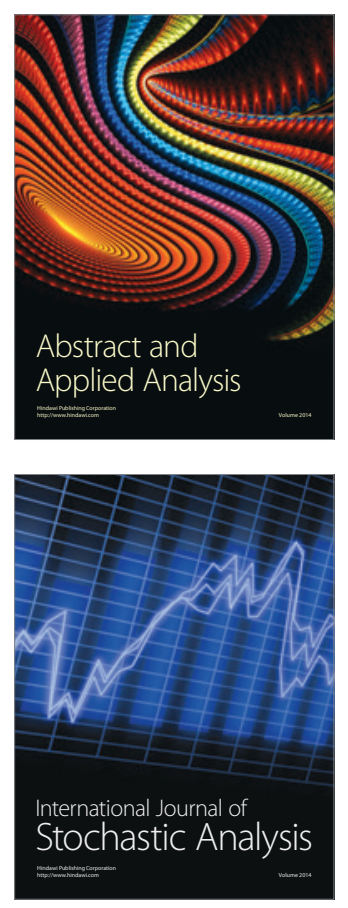

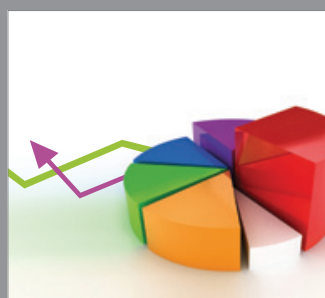

ournal of

Probability and Statistics

Promensencen
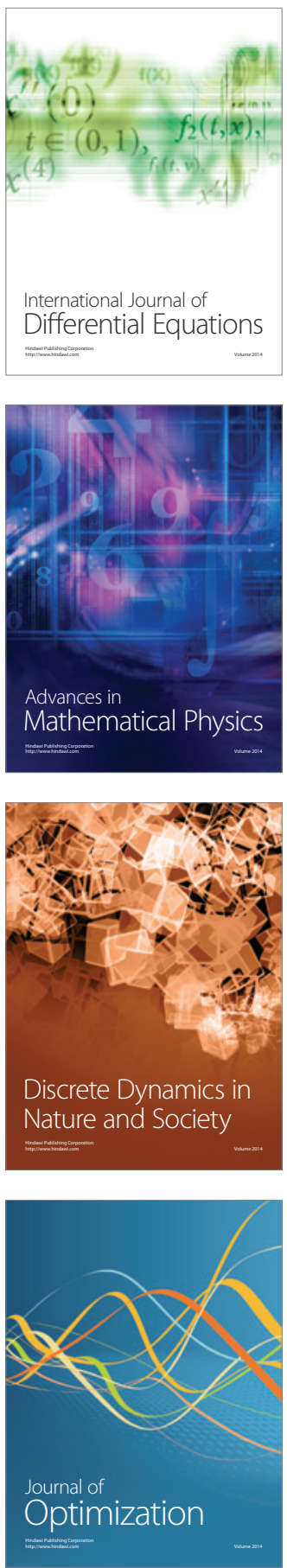